\title{
Moisture Absorption and Desorption of Composite Materials
}

\author{
Chi-Hung Shen and George S. Springer \\ Department of Mechanical Engineering \\ The University of Michigan \\ Ann Arbor, Michigan 48109
}

(Received December 10, 1975)

\begin{abstract}
Expressions are presented for the moisture distribution and the moisture content as a function of time of one dimensional homogeneous and composite materials exposed either on one side or on both sides to humid air or to water. The results apply during both moisture absorption and desorption when the moisture content and the temperature of the environment are constant. Test procedures are described for determining experimentally the values of the moisture content and the diffusivity of composite materials. A series of tests using unidirectional and $\pi / 4 \mathrm{Graphite}$ $\mathrm{T}-300$ Fiberite 1034 composites were performed in the temperature range $300-425 \mathrm{~K}$ with the material submerged both in moist air (humidity 0 to $100 \%)$ and in water. The test data support the analytical results and provide the moisture absorption and desorption characteristics of such composites. Extension of the results to materials exposed to time varying environmental conditions is ind icated.
\end{abstract}

\section{SCOPE}

$\mathbf{O}$ WING TO THEIR favorable performance characteristics composite materials have been gaining wide use in commercial, military, and space applications. There is some concern, however, that the mechanical properties of such materials may suffer when the material is exposed to moisture for long periods of time. Therefore, in order to utilize the full potential of composite materials their response to moist environments must be known. The objective of this investigation was to determine the moisture content as a function of time of composite materials when the material is either exposed to humid air or fully submerged in water. Although the main interest in this investigation was in the moisture absorption and desorption of composite materials, the results also apply to homogeneous materials.

The results presented here apply directly when the moisture content and the temperature of the environment are constant. The results obtained are also needed 
for calculating moisture contents of materials when the ambient conditions vary with time.

\section{CONCLUSION AND SIGNIFICANCE}

The following problem was investigated. A material (either homogeneous or composite) is exposed to a moist environment. Depending upon the environmental conditions and the condition of the material, the material either absorbs or loses moisture as manifested by weight gain or weight loss. The objective is to determine the percent moisture content $M$ (percent weight gain) of the material as a function of time $t$

$$
M=M(t)=\frac{\text { Weight of moist material }- \text { Weight of dry material }}{\text { Weight of dry material }} \times 100
$$

It was found that when a) the material is exposed to the environment on one side only, or on two sides with both sides being parallel (one dimensional problem, Figures $1 \mathrm{a}$ and $1 \mathrm{~b}$ ), b) initially the temperature and moisture distributions inside the material are uniform, and c) the moisture content and the temperature of the environment are constant, the moisture content of the material during both adsorption and desorption is

$$
M=G\left(M_{m}-M_{i}\right)+M_{i}
$$

$M_{i}$ is the initial moisture content of the material, $M_{m}$ is the maximum moisture content which can be attained under the given environmental conditions, and $G$ is a time dependent parameter

$$
G=1-\frac{8}{\pi^{2}} \sum_{j=o}^{\infty} \frac{\exp \left[-(2 j+1)^{2} \pi^{2}\left(\frac{D_{x} t}{s^{2}}\right)\right]}{(2 j+1)^{2}}
$$

Equation (3) may be approximated by the expression (see Figure 2)

$$
G=1-\exp \left[-7.3\left(\frac{D_{x} t}{s^{2}}\right)^{0.75}\right]
$$

For a material exposed on two sides to the same environment $s$ is equal to the thickness $(s=h)$; for a material insulated on one side (thermal insulation, impermeable to moisture) $s$ is twice the thickness $(s=2 h)$. $D_{x}$ is the diffusivity of the material in the direction normal to the surface. For fiber reinforced composites in which the orientations of all the fibers with respect to the $x, y, z$ axes are $\alpha, \beta$, and $\gamma$ (Figure 1) 


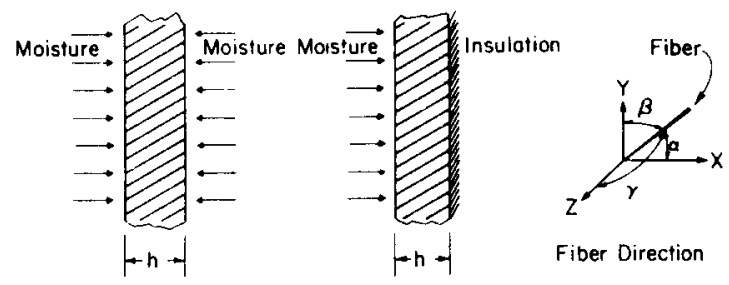

Figure 1. Description of the problem.

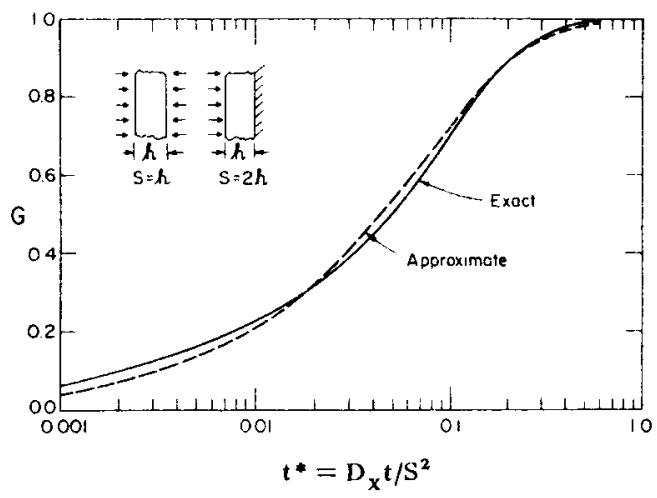

Figure 2. The variation of the parameter $G$ with the dimensionless time $t^{*}=D_{x} t / s^{2}$. Exact:

Equation (3); approximate: Equation (4).

$$
D_{x}=D_{11} \cos ^{2} \alpha+D_{22} \sin ^{2} \alpha
$$

where $D_{11}$ and $D_{22}$ are the diffusivities in the directions parallel and normal to the fibers (longitudinal and transverse directions). If $D_{x}$ is not known it may be estimated from the diffusivity of the matrix $D_{r}$ and the volume fraction of the fibers $v_{f}$

$$
D_{x}=D_{r}\left[\left(1-v_{f}\right) \cos ^{2} \alpha+\left(1-2 \sqrt{v_{f} / \pi}\right) \sin ^{2} \alpha\right]
$$

Equation (6) may be applied to unidirectional and laminated composites (e.g. $\pi / 4$ composites). In the latter case in each laminate a) $D_{r}$ must be the same, b) the volume fraction of the fibers $v_{f}$ must be the same, and c) the fibers must be in a plane parallel to the surface $\left(\alpha=90^{\circ}\right)$. The orientations of the fibers relative to the $y, z$ directions may vary from laminate to laminate.

Experimental evidence indicates that the maximum moisture content is insensitive to the temperature but depends on the moisture content of the environment. For a material immersed in liquid $M_{m}$ is a constant 


$$
M_{m}=\text { constant (liquid) }
$$

For a material exposed to humid air $M_{m}$ depends on the relative humidity $\phi$ according to the relationship

$$
M_{m}=a \phi^{b} \quad \text { (humid air) }
$$

The time required for a material to attain at least 99.9 percent of its maximum possible moisture content is

$$
t_{m}=\frac{0.67 s^{2}}{D_{x}}
$$

The time required to reach the maximum moisture content is insensitive to the moisture content of the environment but depends on the temperature through $D_{x}$.

The foregoing results for $M$ and $t_{m}$ are based on an exact solution of the equations governing the problem (Section III). However, in order to calculate $M$ and $\mathrm{t}_{m}$ the maximum moisture content $M_{m}$ (or the constants $a$ and $b$ ) and the diffusivity $D_{x}$ must be known. These parameters must be determined experimentally. The accuracy of $M$ and $t_{m}$ depend directly on the accuracy of the experiments. Proper test procedures for determining $M_{m}, a, b$, and $D_{x}$ are described in Section V.

An extensive series of tests were performed studying the moisture absorption and desorption characteristics of Graphite T-300 Fiberite 1034 composites. These tests gave the following results:

Graphite T-300 Fiberite 1034 exposed to humid air

$$
M_{m}=0.00014(\phi)^{2} \quad \text { percent }
$$

Graphite T-300 Fiberite 1034 immersed in water

$$
M_{m}=1.8 \quad \text { percent }
$$

The matrix diffusivity together with the longitudinal and transverse diffusivities are shown in Figure 3.

\section{ANALYSIS}

Consider a plate of thickness $h$ exposed on two sides to the same moist environment (Figure 1a). The plate is taken to be infinite in the $y$ and $z$ directions so that the moisture content and the temperature inside the plate vary only in the $x$ 


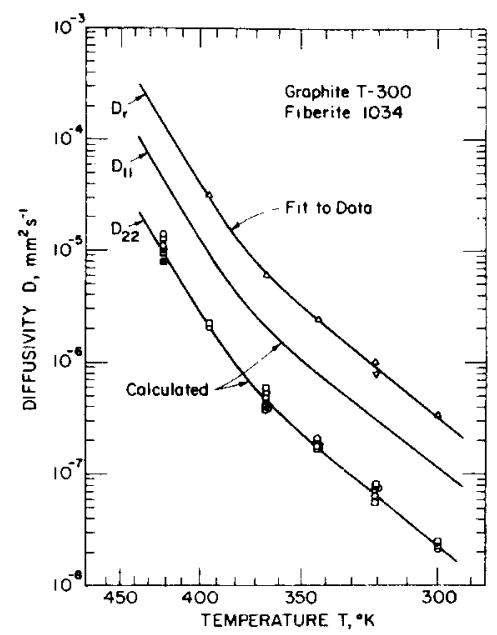

Figure 3. The matrix diffusivity $D_{r}$, the transverse diffusivity $D_{22}$, and the longitudinal diffusivity $D_{11}$ of Graphite T.300 Fiberite 1034 composites. $\left(D_{11}\right.$ and $D_{22}$ are for $v_{f}=0.68$ and $\left.\alpha=90^{\circ}\right) \circ$, * : unidirectional; $\square$, - : $\pi / 4$ (empty and dark symbols represent absorption and desorption, respectively). $\Delta$ : present data for pure matrix; $\nabla$ : data from Hertz [4]. Calculated $D_{1,}$ and $D_{22}$ curves are from Equations (24a and $24 b$ ).

direction (i.e. the problem is one dimensional). Initially (time $t<0$ ) the temperature $T_{i}$ and the moisture concentration $c_{i}$ inside the plate are uniform. The plate is suddenly exposed to a moist environment in which the temperature $T_{a}$ and moisture concentration $c_{a}$ are constant. The objective is to determine the temperature distribution $T$, the moisture distribution $c$, and the total moisture content $m$ of the material as a function of time.

The temperature and the moisture concentration are described by the Fourier and the Fick equations

$$
\begin{gathered}
\rho C \frac{\partial T}{\partial t}=\frac{\partial}{\partial x} K_{x} \frac{\partial T}{\partial x} \\
\frac{\partial c}{\partial t}=\frac{\partial}{\partial x} D_{x} \frac{\partial c}{\partial x^{2}}
\end{gathered}
$$

and the initial and boundary conditions

$$
\begin{array}{llr}
T=T_{i} & o<x<h \quad t \leqslant o \\
c=c_{i} & & \\
T=T_{a} & x=o ; x=h & t>o \\
c=c_{a} & &
\end{array}
$$

The moisture concentration at the surface of the material $c_{m}$ is related to the moisture content of the environment.

The thermal diffusivity $K_{x} / \rho C$ and the mass diffusivity $D_{x}$ depend on the temperature and on the moisture concentration and hence on the distance $x$. The 
parameters $K_{x} / \rho C$ and $D_{x}$ are a measure of the "speed" by which the temperature and the moisture concentration change inside the material. For most materials the ratio $\left(K_{x} / \rho C\right) / D_{x}$ is of the order of $10^{6}$. Thus, the temperature approaches equilibrium about $10^{6}$ times faster than the moisture concentration. For example, for a $12.5 \mathrm{~mm}$ thick Graphite T-300 Fiberite 1034 composite exposed to 90 percent humid air at $350 \mathrm{~K}$ the temperature becomes nearly uniform across the plate in about 15 seconds while the moisture concentration reaches equilibrium only in about 13 years. Therefore, in most practical situations the temperature inside the material may be taken to be the same as the ambient temperature. It has also been observed that the diffusivity changes very little with the moisture content [1]. With the temperature and the diffusivity being constant inside the material the problem is described by the equations

$$
\begin{array}{cc} 
& \frac{\partial c}{\partial t}=D_{x} \frac{\partial^{2} c}{\partial x^{2}} \\
c=c_{i} & o<x<h \quad t \leqslant o \\
c=c_{a} & x=o ; x=h \quad t>o
\end{array}
$$

The solution of the Equations (15) and (16) is given by Jost [2]

$$
\frac{c-c_{i}}{c_{m}-c_{i}}=1-\frac{4}{\pi} \sum_{j=0}^{\infty} \frac{1}{(2 j+1)} \sin \frac{(2 j+1) \pi x}{h} \exp \left[-\frac{(2 j+1)^{2} \pi^{2} D_{x} t}{h^{2}}\right]
$$

The total weight of the moisture in the material is obtained by integrating Equation (17) over the plate thickness

$$
m=\int_{o}^{h} c d x
$$

The result of this integration is [2]

$$
G \equiv \frac{m-m_{i}}{m_{m}-m_{i}}=1-\frac{8}{\pi^{2}} \sum_{j=1}^{\infty} \frac{\exp \left[-(2 j+1)^{2} \pi^{2}\left(\frac{D_{x} t}{h^{2}}\right)\right]}{(2 j+1)^{2}}
$$

$m_{i}$ is the initial weight of the moisture in the material (i.e. the weight prior to exposure to the moist environment) and $m_{m}$ is the weight of moisture in the 
material when the material is fully saturated, in equilibrium with its environment. An analytical approximation for $G$ is given by Equation (4).

In most practical situations we are interested in the percent moisture content as manifested by the weight gain of the material. Thus, in practice the parameter of interest is the percent moisture content defined as

$$
M=\frac{\text { Weight of moist material }- \text { Weight of dry materia! }}{\text { Weight of dry material }} \times 100=\frac{W-W_{d}}{w_{d}} \times 100
$$

By noting that

$$
w=w_{d}+m
$$

Equation (19) may be rearranged in the form

$$
M=G\left(M_{m}-M_{i}\right)+M_{i}
$$

Equation (2) applies to both absorption and desorption. Furthermore, it can be used when the material is exposed to the environment either on one or on two sides. The use of the foregoing results is illustrated by the following examples.

Example 1. A $12.5 \mathrm{~mm}$ thick Graphite T-300 Fiberite 1034 plate is exposed on both sides to air at temperature $350 \mathrm{~K}$ and 90 percent humidity. The plate is of $\pi / 4$ construction with all fibers being parallel to the surface of the plate $\left(\alpha=90^{\circ}\right)$. The initial moisture content of the plate is 0.5 percent. Estimate the time required to reach one percent moisture content. From Equation (4)

$$
t=\frac{s^{2}}{D_{x}}\left[-\frac{1}{7.3} \ln \left(1-\frac{M-M_{i}}{a \phi^{b}-M_{i}}\right)\right]^{1 / 0.75}
$$

For Graphite T-300 Fiberite $1034\left(v_{f}=0.68\right)$ the constants $a$ and $b$ are 0.00014 and 2, respectively. The diffusivity is (Figure 3 and Equation 5)

$$
D_{x}=D_{22}=2.6 \times 1 \sigma^{-7} \mathrm{~mm}^{2} \mathrm{~s}^{-1}
$$

Thus, Equation (21) gives

$$
t=\frac{(12.5)^{2}}{2.6 \times 10^{7}}\left[-\frac{1}{7.3} \ln \left(1-\frac{1-0.5}{0.00014(90)^{2}-0.5}\right)\right]^{\frac{1}{0.75}}=7.6 \times 10^{7} \mathrm{~s}=2.4 \mathrm{yrs} \text {. }
$$




\section{Moisture Absorption and Desorption}

For 90 percent humidity the maximum possible moisture content of the plate is

$$
M_{m}=0.00014(90)^{2}=1.13 \%
$$

The time required to reach at least 99.9 percent of this moisture content is (Equation 9)

$$
t_{m}=\frac{0.67 \times(12.5)^{2}}{2.6 \times 10^{-7}}=4 \times 10^{8} \mathrm{~s}=12.8 \mathrm{yrs}
$$

If the plate were exposed to the environment on one side only the time required to reach one percent moisture content would be

$t=\frac{(2 \times 12.5)^{2}}{2.6 \times 1 \sigma^{7}}\left[-\frac{1}{7.3} \ln \left(1-\frac{1-0.5}{0.00014(90)^{2}-0.5}\right)\right]^{\frac{1}{0.75}}=3.0 \times 10^{8} \mathrm{~s}=9.6 \mathrm{yrs}$

and the time required to reach at least 99.9 percent of the maximum possible moisture content would be

$$
t_{m}=\frac{0.67 \times(2 \times 12.5)^{2}}{2.6 \times 10^{7}}=1.6 \times 10^{9} \mathrm{~s}=51 \mathrm{yrs}
$$

Example 2. The initial moisture content of the plate specified in the above example is 1 percent. The plate is then exposed on both sides to humid air of temperature $333 \mathrm{~K}$ and relative humidity 10 percent. Estimate the moisture content of the plate after 10 days.

From Equation (2)

$$
\begin{aligned}
M= & \left\{1-\exp \left[-7.3\left(\frac{10 \times 24 \times 3600 \times 1.13 \times 10^{-7}}{12.5 \times 12.5}\right)^{0.75}\right]\right\}\left[0.00014(10)^{2}-1\right] \\
& +1=0.97 \%
\end{aligned}
$$

Thus, in 10 days the moisture content would be reduced from one percent to 0.97 percent.

\section{DIFFUSIVITY}

The diffusivity $D_{x}$ can be measured directly. For composite materials the 
measurements must be performed properly taking into consideration the anisotropy of the material. For fiber reinforced composites the diffusion coefficient $D_{x}$ can also be calculated from the following parameters: a) the diffusivity of the matrix $D_{r}$, b) the diffusivity of the fibers $D_{f}$, c) the volume fraction of the fiber $v_{f}$, and d) the orientation of the fibers with respect to the exposed surface $(\alpha, \beta, \gamma$, Figure 1$)$. The relationship between $D_{x}$ and $D_{m}, D_{f}, v_{f}$, and the fiber orientation can be established by noting the similarities between heat conduction and moisture diffusion through the material (Equations 12 and 13). Approximations for the thermal conductivities parallel and normal to the fibers were given by Springer and Tsai [3] $\left(v_{f}<0.785\right)$

$$
\begin{aligned}
K_{11}= & \left(1-v_{f}\right) K_{r}+v_{f} K_{f} \\
K_{22}= & \left(1-2 \sqrt{v_{f} / \pi}\right) K_{r}+\frac{K_{r}}{B_{K}}\left[\pi-\frac{4}{\left.\sqrt{1-\left(B_{K}^{2} v_{f} / \pi\right.}\right)} \tan ^{-1} \frac{\left.\sqrt{1-\left(B_{K}^{2} v_{f} / \pi\right.}\right)}{1+\sqrt{B_{K}^{2} v_{f} / \pi}}\right] \\
& B_{K} \equiv 2\left(\frac{K_{r}}{K_{f}}-1\right)
\end{aligned}
$$

Analogously, we can write

$$
\begin{aligned}
& D_{11}=\left(1-v_{f}\right) D_{r}+v_{f} D_{f} \\
& D_{22}=\left(1-2 \sqrt{v_{f} / \pi}\right) D_{r}+\frac{D_{r}}{B_{D}}\left[\pi-\frac{4}{\sqrt{1-\left(B_{D}^{2} v_{f} / \pi\right)}} \tan ^{-1} \frac{\left.\sqrt{1-\left(B_{D}^{2} v_{f} / \pi\right.}\right)}{1+\sqrt{B_{D}^{2} v_{f} / \pi}}\right] \\
& B_{D} \equiv 2\left(\frac{D_{r}}{D_{f}}-1\right)
\end{aligned}
$$

Generally, the diffusivity of the fiber is small compared to the diffusivity of the matrix $\left(D_{f} \ll D_{r}\right)$, and Equations (23a) and $(23 b)$ reduce to $\left(v_{f}<0.785\right)$

$$
\begin{aligned}
& D_{11}=\left(1-v_{f}\right) D_{r} \\
& D_{22}=\left(1-2 \sqrt{v_{f} / \pi}\right) D_{r}
\end{aligned}
$$

$D_{x}$ is related to $D_{11}, D_{22}$ and $D_{r}$ by Equations (5) and (6).

In order to assess the validity of Equation (6) the diffusivities of Graphite T-300 
Fiberite 1034 composites were measured at different temperatures, and the measured values were compared to values calculated from Equation (6). The tests were performed with $101.6 \mathrm{~mm}$ long, $12.7 \mathrm{~mm}$ wide. and $1.27 \mathrm{~mm}$ thick specimens (volume fraction $v_{f}=0.68$, angle $\alpha=90$ degrees) according to the procedures outlined in the next section. Diffusivities of the pure matrix $D_{r}$ were also measured with $50.8 \mathrm{~mm} \times 12.7 \mathrm{~mm} \times 1.27 \mathrm{~mm}$ specimens. The results for $D_{r}$ are presented in Figure 3. In this figure the data of Hertz [4] is also included.

Comparison between the measured and calculated $D_{x}$ values are shown in Figure 3. As can be seen from this figure there is good correlation between the measured and calculated diffusivities lending confidence to the validity of Equation (6).

\section{TEST PROCEDURES}

In order to predict the moisture content of a material the maximum moisture content $M_{m}$ and the diffusivity $D_{x}$ must be known (see Equations 2,3). The following test procedures may be used to determine these parameters:

1) The test specimen is made in the form of a thin plate $(h / l \ll 1$ and $h / n \ll 1$, Figure 4). so that moisture enters predominantly through the $n l$ surfaces of the plate. A coating impermeable to moisture should be applied to the edges of the plates (surfaces $n h$ and $l h$ ). In the absence of coating a correction must be made for the moisture entering through the edges ("edge effects"). For unidirectional composites the fibers may be oriented in any arbitrary direction; specimens with fibers normal $\left(\alpha=0^{\circ}\right)$ or parallel $\left(\alpha=90^{\circ}\right)$ to the $n l$ surface are most convenient. For laminated composites the fibers in each laminate must be parallel $\left(\alpha=90^{\circ}\right)$ to the nl surface.

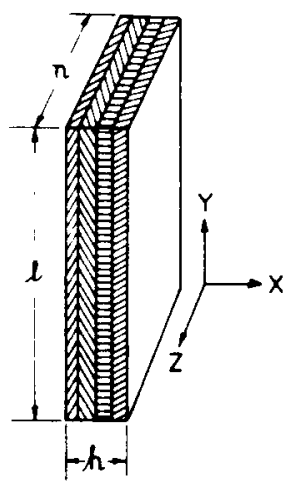

Figure 4. Geometry of the test specimen.

2) The specimen is completely dried in a desiccator and its dry weight $W_{d}$ is measured.

3) The specimen is placed in a constant temperature, constant moisture environment and its weight $W$ is recorded as a function of time.

4) The moisture content (percent weight gain) $M=\left(W-W_{d}\right) / W_{d}$ is plotted versus $\sqrt{t}$, as illustrated in Figure 5.

5) The tests are repeated for different temperatures and different environmental moisture contents.

The above procedure yields a series of curves similar to the one shown in Figure 5.

Initially (when $t<t_{L}$, Figure 5) all curves are straight lines, the slope being proportional to the diffusivity of the material. After a long period of time the curves approach asymptotically the maximum moisture content $M_{m}$. The value of $M_{m}$ is a constant when the material is fully submerged in a liquid; it varies with the 


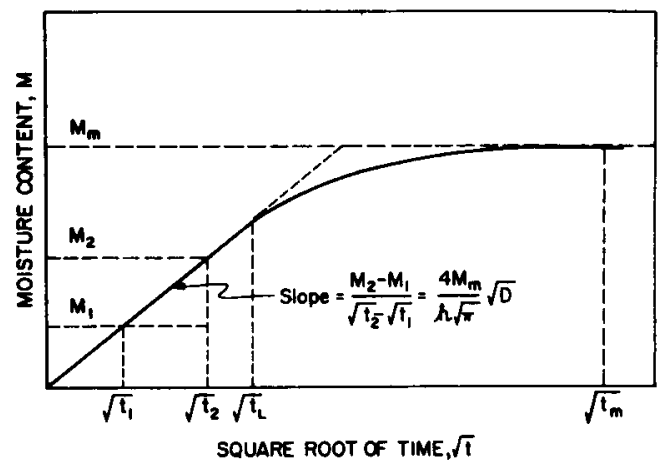

Figure 5. Illustration of the change of moisture content with the square root of time. For $t<t_{L}$ the slope is constant.

relative humidity when the material is exposed to moist air [5]. In the latter case $M_{m}$ may be expressed as

$$
M_{m}=a \phi^{b}
$$

The constants $a$ and $b$ are selected to provide the best fit to the data, as illustrated in the next section.

The diffusivity $D$ is obtained from the initial slope $\left(t<t_{L}\right)$ of the $M_{m}$ versus $\sqrt{t}$ curve (see Appendix)

$$
D=\pi\left(\frac{h}{4 M_{m}}\right)^{2}\left(\frac{M_{2}-M_{1}}{\sqrt{t_{2}}-\sqrt{t_{1}}}\right)^{2}
$$

If the moisture entering the specimen through the "edges" (surfaces $h n$ and $h l$, Figure 6) can be neglected $D_{x}$ is

$$
D_{x}=D
$$

Including "edge effects", for a homogeneous material (Fige: Appendix)

$$
D_{x}=D\left(1+\frac{h}{l}+\frac{h}{n}\right)^{-2}
$$

For fiber reinforced composites $D_{x}$ cannot be computed directly when the edge effects are taken into account. In this case $D_{x}$ must be evaluated from the expressions derived in the Appendix. 


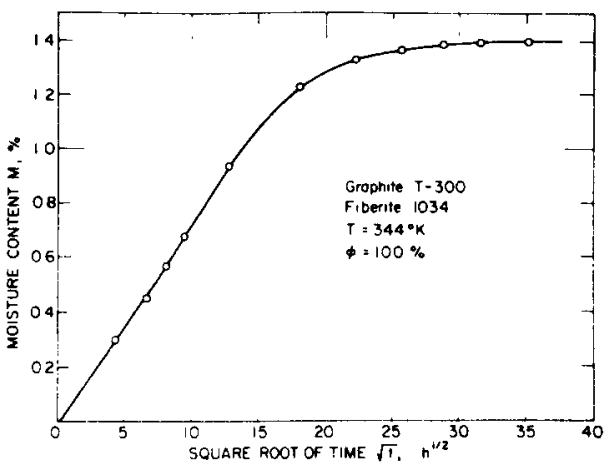

Figure 6. Typical test results for a unidirectional Graphite T-300 Fiberite 1034 composite $\left(v_{f}=0.68\right.$ and $\left.\alpha=90^{\circ}\right)$.

If $D_{r}$ is known then $D_{x}$ can be obtained directly from Equation (6). Hence only $M_{m}$ must be obtained from the tests, in which case, the test times can be reduced by performing the tests at high temperatures.

The foregoing procedure requires that each specimen be tested until the maximum moisture content is reached $\left(t=t_{m}\right)$. The tests may be accelerated by the following procedure.

1) Prepare a specimen in the form of a thin piate as described in point 1 above. The edges must be coated with a substance impermeable to moisture because the edge corrections (given in the Appendix) cannot be applied now.

2) Measure the weight gain of a dry specimen $\left(M_{i}=0\right)$ as a function of time and plot $M$ versus $\sqrt{t}$ beyond the point at which the slope of the curve is not a constant $\left(t>t_{L}\right)$.

3) Assume a value for $D_{x}$.

4) Select a time $t_{1}$ so that $t=t_{1}<t_{L}$.

5) Calculate $G$ from either Equation (3) or from Figure 2.

6) Calculate $M_{m}$ for $t_{1}$ from Equation (2) using the value of $M$ measured at $t_{1}$.

7) Select a time $t_{2}$ so that $t=t_{2}>t_{L}$.

8) Calculate $G$ from either Equation (3) or from Figure 2.

9) Calculate $M_{m}$ for $t_{2}$ from Equation (2) using the value of $M$ measured at $t_{2}$.

10) Check whether $M_{m}$ given by step 6 is equal to $M_{m}$ given by step 9 .

If the two $M_{m}$ values differ select a new diffusivity and repeat the procedure.

Using this procedure the tests can be terminated at a time $t_{2}$ which is much less than $t_{m}$.

\section{RESULTS FOR GRAPHITE T-300 FIBERITE 1034 COMPOSITES}

A series of tests were performed to measure the moisture content of $\pi / 4$ and unidirectional Graphite T-300 Fiberite 1034 composites. The tests were conducted 
with the specimen either exposed to humid air or fully immersed in water. The conditions employed in the tests are summarized in Table 1. Four unidirectional and four $\pi / 4$ specimens were tested at each of the 16 test conditions. Thus a total of 128 specimens were tested. A typical test result is shown in Figure 6. Presentation of all the results in such a manner would require 128 similar plots. However, the accuracy of the tests can be assessed by calculating $G$ from the data (see Equation 2) and by comparing the values obtained with the theoretical $G$ values given in Figure 2. This comparison, shown in Figure 7, shows good agreement between the data and the theory.

Table 1. Summary of Conditions Used in the Tests with Unidirectional and $\pi / 4$ Graphite T-300 Fiberite 1034 Composites. For all Specimens Fiber Volume Fraction $v_{f}=0.65$ to 0.68 ; Fiber Orientation $\alpha=90^{\circ}$

\begin{tabular}{rcccccccc}
\hline Moisture Content & \multicolumn{5}{c}{ Temperature $K$} & \multirow{2}{*}{ Remarks } \\
\hline Humid air 100\% & 300 & 322 & 344 & 366 & - & - & Absorption \\
$75 \%$ & 300 & 322 & 344 & 366 & - & - & Absorption \\
$50 \%$ & - & 322 & 344 & 366 & - & - & Absorption \\
$0 \%$ & - & - & - & 366 & - & 422 & Desorption \\
Saturated steam & - & - & - & - & 394 & 422 & Absorption \\
Immersed in water & - & - & 344 & 366 & - & 422 & Absorption \\
\hline
\end{tabular}

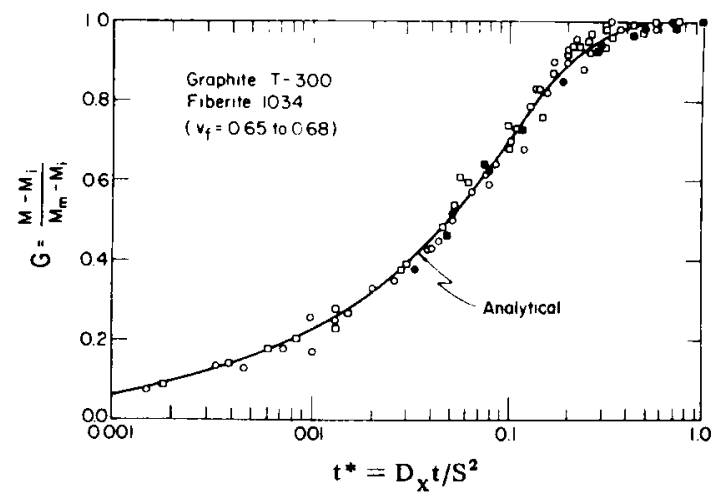

Figure 7. Comparison of analytical (Equation 3) and measured $G$ values for unidirectional and $\pi / 4$ Graphite T-300 Fiberite 1034 composites $\left(v_{f}=0.68\right.$ and $\left.\alpha=90^{\circ}\right) . \circ, \bullet:$ unidirectional; ㅁ. : $\pi / 4$ (empty and dark symbols represent absorption and desorption, respectively). 
The maximum moisture content as a function of humidity is shown in Figure 8. A fit to the data gives

$$
M_{m}=0.00014(\phi)^{2} \quad \text { percent }
$$

For the material fully submerged in water the tests give

$$
M_{m}=1.8 \quad \text { percent }
$$

Equations (10) and (11) apply to both unidirectional and $\pi / 4$ composites.

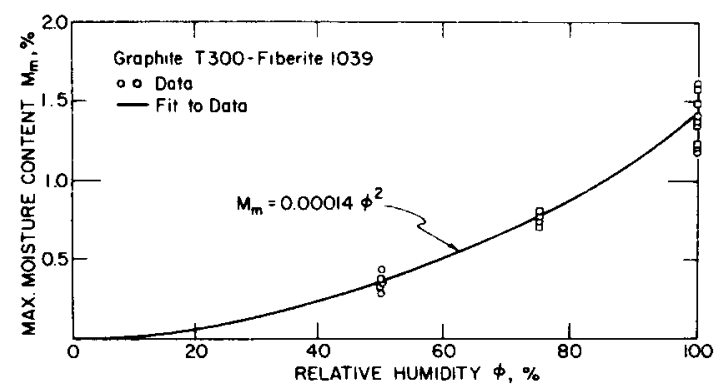

Figure 8. The variation of the maximum moisture content with relative humidity for Graphite $T-300$ Fiberite 1034 composites. $\left(v_{f}=0.68\right.$ and $\left.\alpha=90^{\circ}\right)$ o: unidirectional; $\square: \pi / 4 ;-$ fit to

data,

\section{TIME VARYING BOUNDARY CONDITIONS}

The moisture content given by Equation (2) is applicable only when the initial temperature and moisture concentration inside the material are uniform, and the temperature and moisture content of the environment are constant. When these conditions are not met and when, in addition, the conditions on the two sides of the plates differ the solution to the problem must be obtained by numerical means. A computer code for such general problems has been developed at The University of Michigan and may be obtained from the Fluid Dynamics Laboratory, Department of Mechanical Engineering, The University of Michigan.

\section{ACKNOWLEDGMENTS}

This work was supported by the United States Air Force Materials Laboratory, Air Force Systems Command, Wright-Patterson Air Force Base, Dayton, Ohio under contract number F33615-75-C-5765. 


\section{NOMENCLATURE}

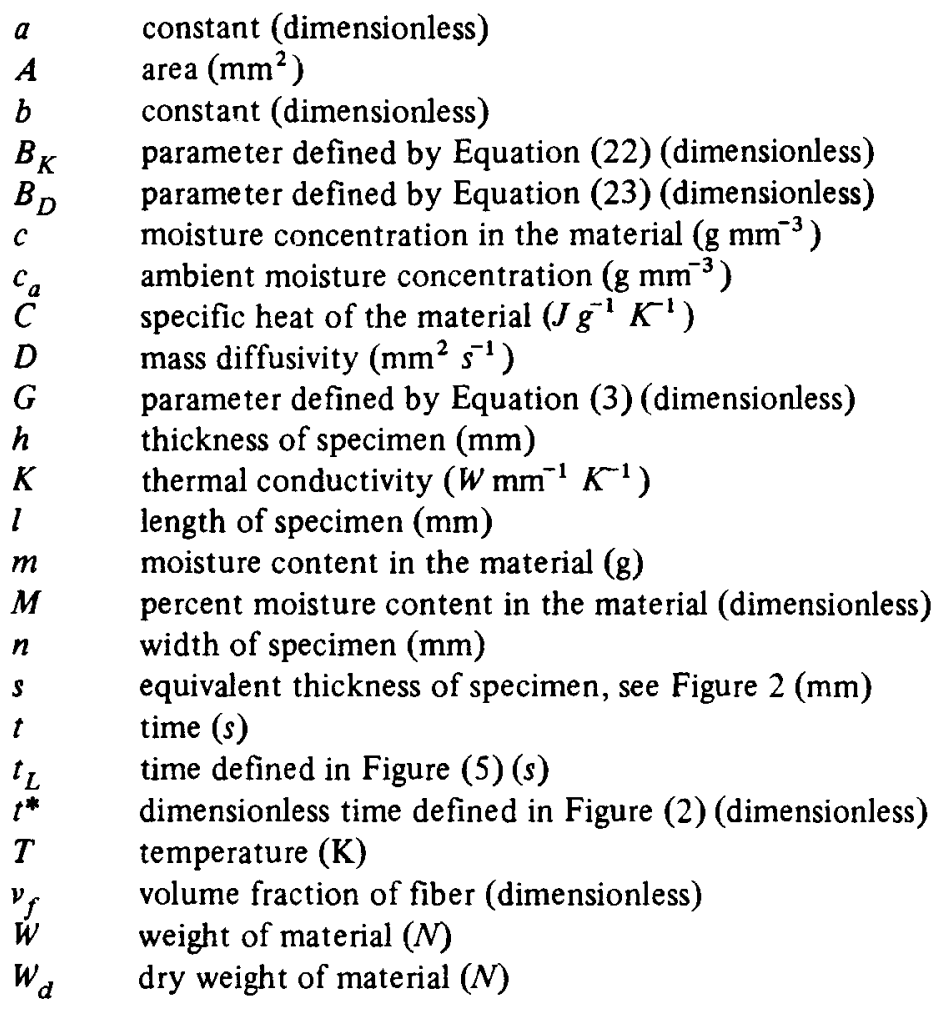

\section{Greek letters}

$\alpha, \beta, \gamma \quad$ fiber orientation with respect to the $x, y$, and $z$ axes, see Figure 1 (rad)

$\phi \quad$ percent relative humidity (dimensionless)

$\rho$ density of material $\left(\mathrm{g} \mathrm{mm}^{-3}\right)$

\section{Subscripts}

$\begin{array}{ll}f & \text { fiber } \\ i & \text { initial state } \\ m & \text { maximum saturation } \\ r & \text { matrix } \\ x, y, z & \text { in the } x, y, \text { and } z \text { directions } \\ 11 & \text { in the direction parallel to the fiber } \\ 22 & \text { in the direction normal to the fiber }\end{array}$




\section{APPENDIX}

\section{Corrections for Edge Effects}

To determine the diffusivity $D_{x}$ we refer to Equation (15)

$$
\frac{\partial c}{\partial t}=D_{x} \frac{\partial^{2} c}{\partial x^{2}}
$$

For a semi-infinite solid $(h \rightarrow \infty)$ the boundary conditions (Equations 16a and 16b) become

$$
\begin{array}{lll}
c=c_{i} & o<x<\infty & t \leqslant o \\
c=c_{m} & x=o & t>o
\end{array}
$$

In this case the concentration as a function of position and time in the solid is [2]

$$
\frac{c-c_{i}}{c_{m}-c_{i}}=1-\operatorname{erf} \frac{x}{2 \sqrt{D_{x} t}}
$$

and the weight of moisture which enters or leaves through an area $A$ in time $t$ is

$$
m=\int_{o}^{t}-A D_{x}\left(\frac{\partial c}{\partial x}\right)_{x=o} d t
$$

Equations (A2) and (A3) give [2]

$$
m=2 A\left(c_{m}-c_{i}\right) \sqrt{\frac{D_{x} t}{\pi}}
$$

During a test moisture enters or leaves the specimen on all six sides (Figure 4). In the early stages of the process the interaction of the different sides may be neglected and Equation (A4) can be applied to each side independently. Thus the total weight of the entering moisture is

$$
m=4\left(c_{m}-c_{i}\right)\left(n l \sqrt{D_{x}}+n h \sqrt{D_{y}}+h l \sqrt{D_{z}}\right) \sqrt{\frac{t}{\pi}}
$$


where $D_{x}, D_{y}$, and $D_{z}$ are the diffusivities in the $x, y$, and $z$ directions. For an initially dry specimen $\left[c_{i}=0\right.$ and $\left.W_{d}=(\rho)(\mathrm{g})(h n l)\right]$ the moisture content as a function of time is

$$
M=\frac{W-W_{d}}{W_{d}} \times 100=\frac{m}{W_{d}} \times 100=\frac{4 c_{m}}{\rho \mathrm{g} h}\left(\sqrt{D_{x}}+\frac{h}{l} \sqrt{D_{y}}+\frac{h}{n} \sqrt{D_{z}}\right) \sqrt{\frac{t}{\pi}}
$$

Note that $c_{m} / \rho \mathrm{g}$ is the maximum moisture content

$$
M_{m}=\frac{W_{m}-W_{d}}{W_{d}} \times 100=\frac{m_{m} /(h n l)}{W_{d} /(h n l)} \times 100=\frac{c_{m}}{\rho \mathrm{g}}
$$

Hence

$$
M=\frac{4 M_{m}}{h \sqrt{\pi}}\left(\sqrt{D_{x}}+\frac{h}{l} \sqrt{D_{y}}+\frac{h}{n} \sqrt{D_{z}}\right) \sqrt{t}
$$

Equation (33) shows that $M$ varies linearly with $\sqrt{t}$ at least for a short time after exposure to the environment, i.e. before the opposing sides begin to affect significantly the moisture content. Equation (A8) may be written as

$$
M=\frac{4 M_{m}}{h} \sqrt{\frac{t}{\pi}} \sqrt{D}
$$

where

$$
D=D_{x}\left(1+\frac{h}{l} \sqrt{\frac{D_{y}}{D_{x}}}+\frac{h}{n} \sqrt{\frac{D_{z}}{D_{x}}}\right)^{2}
$$

For a homogeneous material $D_{x}=D_{y}=D_{z}$ and Equation (A10) becomes

$$
D=D_{x}\left(1+\frac{h}{l}+\frac{h}{n}\right)^{2}
$$

For composites the diffusivities $D_{x}, D_{y}$, and $D_{z}$ must first be evaluated.

For unidirectional composites (fiber orientations $\alpha, \beta$, and $\gamma$, Figure 1), the diffusivities are [6]

$$
D_{x}=D_{11} \cos ^{2} \alpha+D_{22} \sin ^{2} \alpha
$$




$$
\begin{aligned}
& D_{y}=D_{11} \cos ^{2} \beta+D_{22} \sin ^{2} \beta \\
& D_{z}=D_{11} \cos ^{2} \gamma+D_{22} \sin ^{2} \gamma
\end{aligned}
$$

Equations (24) and (A12) together with Equation (A10) give $D$ for unidirectional composites

$$
\begin{aligned}
D= & D_{r}\left[\left(1-v_{f}\right) \cos ^{2} \alpha+\left(1-2 \sqrt{v_{f} / \pi}\right) \sin ^{2} \alpha\right] \\
& {\left[1+\frac{h}{l} \sqrt{\frac{\left(1-v_{f}\right) \cos ^{2} \beta+\left(1-2 \sqrt{v_{f} / \pi}\right) \sin ^{2} \beta}{\left(1-v_{f}\right) \cos ^{2} \alpha+\left(1-2 \sqrt{v_{f} / \pi}\right) \sin ^{2} \alpha}}\right.} \\
& \left.+\frac{h}{n} \sqrt{\frac{\left(1-v_{f}\right) \cos ^{2} \gamma+\left(1-2 \sqrt{v_{f} / \pi}\right) \sin ^{2} \gamma}{\left(1-v_{f}\right) \cos ^{2} \alpha+\left(1-2 \sqrt{v_{f} / \pi}\right) \sin ^{2} \alpha}}\right]^{2}
\end{aligned}
$$

We consider next laminated composites consisting of $N$ layers. The thickness of the $j$ th layer is $h_{j}$. In the $j$ th layer the fiber orientations with respect to the $y$ and $z$ axes are $\beta_{j}$ and $\gamma_{j}$. The fiber orientation with respect to the $x$ axis is $90^{\circ}$ in every laminate, and $\gamma_{j}=90^{\circ}-\beta_{j}$. For these conditions Equation (A12) gives

$$
D_{x}=D_{22}
$$

$$
D_{y}=D_{11} \frac{\sum_{j=1}^{N} h_{j} \cos ^{2} \beta_{j}}{\sum_{j=1}^{N} h_{j}}+D_{22} \frac{\sum_{j=1}^{N} h_{j} \sin ^{2} \beta_{j}}{\sum_{j=1}^{N} h_{j}}
$$

$$
D_{z}=D_{11} \frac{\sum_{j=1}^{N} h_{j} \sin ^{2} \beta_{j}}{\sum_{j=1}^{N} h_{j}}+D_{22} \frac{\sum_{j=1}^{N} h_{j} \cos ^{2} \beta_{j}}{\sum_{j=1}^{N} h_{j}}
$$


Combining Equations (24), (A10), and (A14), we obtain an expression for $D$ for laminated composites

$$
D=D_{r}\left(1-2 \sqrt{v_{f} / \pi}\right)\left[1+\frac{h}{l} \sqrt{\frac{\left(1-v_{f}\right) \sum_{i=1}^{N} h_{j} \cos ^{2} \beta_{j}}{\left(1-2 \sqrt{v_{f} / \pi}\right) \sum_{j=1}^{N} h_{j}}+\frac{\sum_{j=1}^{N} h_{j} \sin ^{2} \beta_{j}}{\sum_{j=1}^{N} h_{j}}}\right.
$$

$$
\left.+\frac{h}{n} \sqrt{\frac{\left(1-v_{f}\right) \sum_{j=1}^{N} h_{j} \sin ^{2} \beta_{j}}{\left(1-2 \sqrt{v_{f} / \pi}\right) \sum_{j=1}^{N} h_{j}}+\frac{\sum_{j=1}^{N} h_{j} \cos ^{2} \beta_{j}}{\sum_{j=1}^{N} h_{j}}}\right]^{2}
$$

\section{REFERENCES}

1. J. M. Augl and R. Trabocco, "Environmental Degradation Studies on Carbon Fiber Reinforced Epoxies," Presented at the "Workshop on Durability of Composite Materials," held on September 30-October 2, 1975 at the Battelle Memorial Institute, Columbus, Ohio.

2. W. Jost, Diffusion in Solids, Liquids, Gases, Academic Press (1960).

3. G. S. Springer and S. W. Tsai, "Thermal Conductivities of Unidirectional Materials," J. Composite Materials, Vol, 1 (1967), p. 166.

4. J. Hertz, "Investigation into the High-Temperature Strength Degradation of Fiber-Reinforced Resin Composite During Ambient Aging," Materials Research Group, Convair Aerospace Division, General Dynamics Corporation, Report No. GDCA-DBG71-004-3, contract NAS 8-27435, March 1972.

5. J. F. Carpenter, "Moisture Sensitivity of Epoxy Composites and Structural Adhesives," McDonnell Aircraft Company, Report MDC A2640, December 1973.

6. H. S. Carslaw and J. G. Jaeger, Conduction of Heat in Solids, Oxford Press (1959). 